\title{
Identification of bird species and their conservation status in Tuti Island, Sudan
}

\author{
Mutasim E. A ${ }^{1}$, Tahani A. $\mathbf{H}^{2}$, Nasir Y. G ${ }^{3}$, Moneer S. $\mathbf{M}^{4}$ \\ ${ }^{1}$ Mutasim Essa Abdallah, Higher council of environment, urban and rural promotion \\ ${ }^{2}$ Tahani Ali Hassan, College of Natural Resources and Environmental Studies, University of Bahri, Sudan. \\ ${ }^{3}$ Nasir Yousif Gaboush, Wildlife Research Center, Sudan \\ ${ }^{4}$ Moneer Salih KhaliL Winji Tour Company, Khartoum, Sudan
}

How to cite this paper: Mutasim, E.A,
Tahani A. H, Nasir Y.G, Moneer S.M. (2019)
Identification of bird species and their
conservation status in Tuti Island, Sudan.
International Journal of Food Science and
Agriculture, 3(4), 257-266.

DOI: $10.26855 /$ ijfsa.2019.12.001

*Corresponding author: Tahani Ali Hassan, Tahani Ali Hassan, College of Natural Resources and Environmental Studies, University of Bahri, Sudan.

Email: tahanihassanm@gmail.com

\begin{abstract}
This study was conducted in Tuti island at the confluence of the White Nile and the Blue Nile in Khartoum city, directly opposite the northern side of the city of Khartoum, the capital of the Sudan, in the period from June 2016 to May 2017 covering three seasons namely cool winter, dry summer and the rainy season, A direct bird's count method is used for data collection. In total, about 8888 individuals that belonged to 88 species, 20 orders were observed in this study.
\end{abstract}

\section{Keywords}

Tuti Island, bird species, direct bird count method

\section{Introduction}

birds are a useful indicator of habitats' health and can be an efficient and straightforward means for either monitoring environmental changes (Furness et al, 1993) or for assessing biodiversity importance (Starkley et al., 2002) as they are taxonomically stable, easily surveyed and wildly distributed across almost all habitats, amongst other reasons. Birds are the easiest group to monitor if changes in forest biodiversity need to be assessed (Benum, 1999). The avifauna of Sudan numbers 937 species of which 616 are resident and 312 are regular seasonal migrants, including 214 Palearctic species.

Data on conservation and protecting of bird populations have been particularly challenging, this is because of the vast range of habitats they occupy during the course of their seasonal cycle and the need to undertake work in different ecological conditions in the feeding and breeding grounds.

The vegetation coverage of the study area is threaten by several human activities including overgrazing and agriculture, change of land use in favor of built forms aggravated by the bridge erection and increasing population (Eltayeb et al. 2012) this is one of factors effecting the birds diversity too.

The Island is stressed by overgrazing and waste disposal, the most important threat, however, is the reclamation of the Island for construction purposes. As the Tuti Island population continues to grow, it is expected that the threats facing the Island will increase in magnitude, unless adequate management plans are designed and thoroughly implemented. There is also a lack of awareness of the importance of the birds and the biodiversity in island by the community (visitors, farmers, fisheries, grazers, etc) and this constitutes a main threat to the habitat.

Reliable information gathered on bird's diversity is useful to identify and conserve the bird's island; such knowledge provides both guidelines for accomplishing the desired conservation strategy. The main objectives of the Study were to identify birds species and their conservation status in Tuti Island. 


\section{Study Area}

Tuti is an Island at the confluence of the White Nile and the Blue Nile in Khartoum city, directly opposite the northern side of the city of Khartoum, the capital of the Sudan. It can be described as a rural "eye" in the center of Khartoum conurbation (Davies, 1994). This feature makes it unique and generates the island exceptional identity to the extended to be named as a "Surprising Place" (Ibrahim \& Davies, 1991).

The total area of the Island in 2012 is about 472.3 Hectare. $\left(15^{\circ} 37^{\prime} \mathrm{N}, 32^{\circ} 31^{\prime} \mathrm{E}\right)$, Tuti is generally flat terrain with gentle slope towards the edges. The southeast part is slightly lower than the other parts, thus it is frequently covered with yearly floods. A recent study on the flora in Tuti island suggested that the island is favored with rich vegetation content (Abdelmageed, 2008). the flora In Tuti Island is much more diverse and complex, containing variable trees, shrubs, grasses and herbs, this difference implies that the Island have a wider range of microhabitats for different species of birds and hence the higher species richness (Abdelmageed, 2008). No previous studies has been conducted on birds in Tuti island so the goal of this study was to determine bird species and their conservation status in Tuti island, Sudan.

\section{Methodology}

The survey was carried out in the period from June 2016 to May 2017, covering three seasons namely cool winter, dry summer and the rainy season. The survey conducted at morning considered the suitable time of the day, and record standards such as birds species, numbers, weather conditions and time. The survey was carried by a team of three ornithologists and one photographer. A direct bird's count method is used for data collection. Bird counts and identification surveys were conducted twice a month for three seasons. The information gathered analyzed and designed in order to generate results that meet the objectives of the study.

\section{Equipments used for data collection:}

- A pair of binoculars (50X50 magnifications).

- Telescope - Swaroski (660 - 940).

- Digital still camera

- Data recording sheets

- Note book

- An identification field guide to the birds of Africa, second edition 2010.

\section{Results and Discussion}

In total, about 8888 individuals that belonged to 88 species, 20 orders were observed in this study (Table 1) and (fig 1- fig 20) . of these, 2 species were in the order Ciconiiformes, 4 species Columbiformes, 5 species were in the order Coraciiformes, 3 species were in the order Apodiformes, 11 species were in the order Pelecaniformes, 31 Passeriformes species were in the order, 13 species were in the order Charadriiformes, 1 species was in the order Accipitriformes, 1 species was in the order Coliiformes, 2 species were in the order Cuculiformes, 2 species were in the order Apodiformes, 3 species were in the order Bucerotiformes, 1 species was in the order Caprimulgiformes, 1 species was in the order Accipitriformes, 1 species was in the order Piciformes, 2 species were in the order Coraciiformes, 2 species were in the order Bucerotiformes, 1 species was in the order Suliformes, 1 species was in the order Anseriformes, 3 species were in the order Coraciiformes.

Generally bird survey results can be strongly influenced by season, time of day and local habitat variation, including elevation (Davies, 2002). Other variables such as weather conditions, human presence, observer's experience, number of observers and observers' own limitations and the fact that the avifauna of Sudan includes a total of 1013 species some of them classified as migratory bird species that relocate during the year can also influence on the presence of birds. Therefore, and for a better understanding or even monitoring of habitat biodiversity, surveys should ideally be done in different seasons and conditions.

The four most common birds identified were the House Sparrow (Passer domesticus) with 2694 birds identified, followed by the Laughing Dove (Spilopelia senegalensis) with 645 birds identified, the Cattle Egret (Bubulcus ibis) with 573 birds identified and the black Greater blue eared starling (Lamprotornis chalybaeus) with 402 birds identified. 
Table 1. Bird species encountered in Tuti Island and their conservation status during 2016- 2017

\begin{tabular}{|c|c|c|c|c|c|}
\hline Order & Family & Species common name & Scientific name & Migratory status & Status/IUCN \\
\hline Ciconiiformes & Ciconiidae & Abdim's Stork & Ciconia abdimii & $\mathrm{AM}$ & $\mathrm{LC}$ \\
\hline Coraciiformes & Coraciidae & Abssiniannian roller & Coracias abyssinicus & $\mathrm{AM} / \mathrm{LM}$ & $\mathrm{LC}$ \\
\hline Columbiformes & Columbidae & African Mourning Dove & Streptopelia decipiens & $\mathrm{R}$ & $\mathrm{LC}$ \\
\hline Apodiformes & Apodidae & African Palm swift & Cypsiurus parvus & $\mathrm{LM} / \mathrm{R}$ & $\mathrm{LC}$ \\
\hline Pelecaniformes & Threskiornithidae & African sacred ibes & Threskiornis aethiopicus & $\mathrm{AM} / \mathrm{LM}$ & $\mathrm{LC}$ \\
\hline Passeriformes & Estrildidae & African Silverbill & Euodice cantans & $\mathrm{R} / \mathrm{LM}$ & $\mathrm{LC}$ \\
\hline Pelecaniformes & Threskiornithidae & African spoon bill & Platalea alba & $\mathrm{AM} / \mathrm{LM}$ & $\mathrm{LC}$ \\
\hline Passeriformes & Nectariniidae & Beautiful sunbird & Cinnyris pulchella & $\mathrm{R}$ & $\mathrm{LC}$ \\
\hline Passeriformes & Muscicapidae & Black Scrub Robin & Cercotrichas podobe & $\mathrm{R}$ & $\mathrm{LC}$ \\
\hline Charadriiformes & Scolopacidae & Black tailled Godwit & Limosa limosa & $\mathrm{PM}$ & $\mathrm{LC}$ \\
\hline Columbiformes & Columbidae & Black-billed Wood Dove & Turtur abyssinicus & $\mathrm{LM} / \mathrm{R}$ & $\mathrm{LC}$ \\
\hline Pelecaniformes & Ardeidae & Black-Headed Heron & Ardea melanocephala & $\mathrm{LM} / \mathrm{R}$ & $\mathrm{LC}$ \\
\hline Charadriiformes & Charadriidae & Black-headed lapwing & Vanellus tectus & $\mathrm{R} / \mathrm{LM}$ & $\mathrm{LC}$ \\
\hline Charadriiformes & Recurvirostridae & Black-winged stilt & Himantopus himantopus & $\mathrm{AM} / \mathrm{PM}$ & $\mathrm{LC}$ \\
\hline Accipitriformes & Accipitridae & Blak kite & Milvus migrans & PM & $\mathrm{LC}$ \\
\hline Coliiformes & Coliidae & Blue-naped Mousebird & Urocolius macrourus & $\mathrm{R}$ & $\mathrm{LC}$ \\
\hline Charadriiformes & Laridae & Caspian tern & Hydroprogne caspia & $\mathrm{PM} / \mathrm{R} / \mathrm{LM}$ & $\mathrm{LC}$ \\
\hline Pelecaniformes & Ardeidae & Cattle Egret & Bubulcusibis & $\mathrm{AM} / \mathrm{LM} / \mathrm{PM}$ & $\mathrm{LC}$ \\
\hline Passeriformes & Pycnonotidae & Common Bulbul & Pycnonotus barbatus & $\mathrm{R}$ & $\mathrm{LC}$ \\
\hline Cuculiformes & Cuculidae & African cuackoo & Cuculus gularis & $\mathrm{AM}$ & $\mathrm{LC}$ \\
\hline Charadriiformes & Charadriidae & Common ringed plover & Charadrius hiaticula & PM & $\mathrm{LC}$ \\
\hline Charadriiformes & Scolopacidae & Common sandpiper & Actitis hypoleucos & $\mathrm{PM}$ & $\mathrm{LC}$ \\
\hline Apodiformes & Apodidae & Common swift & Apus apus & $\mathrm{R} / \mathrm{LM}$ & $\mathrm{LC}$ \\
\hline Passeriformes & Alaudidae & Crested Lark & Galerida cristata & $\mathrm{R}$ & $\mathrm{LC}$ \\
\hline Passeriformes & Estrildidae & Crimson rumped waxbill & Estrilda rhodopyga & $\mathrm{R}$ & $\mathrm{LC}$ \\
\hline Passeriformes & Estrildidae & Cut-throat finch & Amadina fasciata & $\mathrm{R} / \mathrm{LM}$ & $\mathrm{LC}$ \\
\hline Passeriformes & Turdidae & Desert wheatear & Oenanthe deserti & $\mathrm{PM}$ & $\mathrm{LC}$ \\
\hline Charadriiformes & Glareolidae & Egyptian Plover & Pluvianus aegyptius & $\mathrm{LM} / \mathrm{R}$ & $\mathrm{LC}$ \\
\hline Passeriformes & Hirundinidae & Ethiopian Swallow & Hirundo aethiopica & $\mathrm{AM} / \mathrm{R}$ & $\mathrm{LC}$ \\
\hline Bucerotiformes & Upupidae & Eurasian hoopoe & Upupa epops & $\mathrm{AM} / \mathrm{LM} / \mathrm{R}$ & $\mathrm{LC}$ \\
\hline Caprimulgiformes & & Eurasian Nihgt Gare & Caprimulgus europaeus & $\mathrm{PM}$ & $\mathrm{LC}$ \\
\hline Charadriiformes & Burhinidae & Eurasian thick-knee & Burhinus oedicnemus & PM & $\mathrm{LC}$ \\
\hline Accipitriformes & Accipitridae & Gabar Goshawk & Micronisus gabar & $\mathrm{R}$ & $\mathrm{LC}$ \\
\hline Passeriformes & Passeridae & Sudan golden Sparrow & Passer luteus & $\mathrm{LM} / \mathrm{R}$ & $\mathrm{LC}$ \\
\hline Passeriformes & Cisticolidae & Graceful Prinia & Prinia gracilis & $\mathrm{R}$ & $\mathrm{LC}$ \\
\hline Pelecaniformes & Ardeidae & Great egreat & Ardea alba & $\mathrm{PM} / \mathrm{LM} / \mathrm{AM}$ & $\mathrm{LC}$ \\
\hline Pelecaniformes & Pelecanidae & Great white pelican & Pelecanus onocrotalus & $\mathrm{AM} / \mathrm{PM}$ & $\mathrm{LC}$ \\
\hline Passeriformes & Sturnidae & $\begin{array}{l}\text { Greater blue-eared } \\
\text { starling }\end{array}$ & Lamprotornis chalybaeus & $\mathrm{R} / \mathrm{LM}$ & $\mathrm{LC}$ \\
\hline
\end{tabular}


Table 1. cont.

\begin{tabular}{|c|c|c|c|c|c|}
\hline Order & Family & Species common name & Scientific name & Migratory status & Status/IUCN \\
\hline Psittaciformes & Psittacidae & Rose-ringed parakeet & Psittacula krameri & $\mathrm{AM}$ & $\mathrm{LC}$ \\
\hline Charadriiformes & Scolopacidae & Green sandpiper & Tringa ochropus & PM & $\mathrm{LC}$ \\
\hline Pelecaniformes & Ardeidae & Green-backed heron & Butorides striata & & $\mathrm{LC}$ \\
\hline Pelecaniformes & Ardeidae & Grey heron & Ardea cinerea & $\mathrm{LM} / \mathrm{PM}$ & $\mathrm{LC}$ \\
\hline Piciformes & Picidae & Grey woodpecker & Dendropicos goertae & $\mathrm{R}$ & $\mathrm{LC}$ \\
\hline Passeriformes & Passeridae & House Sparrow & Passer domesticus & $\mathrm{R}$ & $\mathrm{LC}$ \\
\hline Columbiformes & Columbidae & Laughing Dove & Spilopelia senegalensis & $\mathrm{LM} / \mathrm{R} / \mathrm{PM}$ & $\mathrm{LC}$ \\
\hline Coraciiformes & Meropidae & Little Bee eater & Merops pusillus & $\mathrm{LM} / \mathrm{R}$ & $\mathrm{LC}$ \\
\hline Pelecaniformes & Ardeidae & Little egreat & Egretta garzetta & $\mathrm{LM} / \mathrm{PM}$ & $\mathrm{LC}$ \\
\hline Charadriiformes & Scolopacidae & Little stint & Calidris minuta & $\mathrm{PM}$ & $\mathrm{LC}$ \\
\hline Apodiformes & Apodidae & African palm swift & Cypsiurus parvus & $\mathrm{R} / \mathrm{LM}$ & $\mathrm{LC}$ \\
\hline Coraciiformes & Alcedinidae & Malachite Kingfisher & Corythornis cristatus & $\mathrm{R} / \mathrm{LM}$ & $\mathrm{LC}$ \\
\hline Charadriiformes & Scolopacidae & Marsh sandpiper & Tringa stagnatilis & PM & $\mathrm{LC}$ \\
\hline Passeriformes & Laniidae & Masked shrik & Lanius nubicus & $\mathrm{PM}$ & $\mathrm{LC}$ \\
\hline Passeriformes & Ploceidae & Northern Masked waver & Ploceus taeniopterus & $\mathrm{LM} / \mathrm{R}$ & $\mathrm{LC}$ \\
\hline Columbiformes & Columbidae & Namaqua Dove & Oena capensis & $\mathrm{R} / \mathrm{LM} / \mathrm{AM} / \mathrm{PM}$ & $\mathrm{LC}$ \\
\hline Passeriformes & Nectariniidae & Nile Valy sunbirds & Hedydipna metallica & $\mathrm{LM} / \mathrm{R}$ & $\mathrm{LC}$ \\
\hline Passeriformes & Ploceidae & Northern red bishop & Euplectes franciscanus & $\mathrm{LM} / \mathrm{R}$ & $\mathrm{LC}$ \\
\hline Passeriformes & Turdidae & Northern wheatear & Oenanthe oenanthe & $\mathrm{PM}$ & $\mathrm{LC}$ \\
\hline Coraciiformes & Alcedinidae & Pied Kingfisher & Ceryle rudis & $\mathrm{R} / \mathrm{LM} / \mathrm{AM}$ & $\mathrm{LC}$ \\
\hline Passeriformes & Muscicapidae & Pied wheatear & Oenanthe pleschanka & $\mathrm{PM}$ & $\mathrm{LC}$ \\
\hline Passeriformes & Viduidae & Pin-tailed Whydah & Vidua macroura & $\mathrm{R}$ & $\mathrm{LC}$ \\
\hline Bucerotiformes & Bucerotidae & $\begin{array}{l}\text { Northern red-billed } \\
\text { hornbill }\end{array}$ & Tockus erythrorhynchus & $\mathrm{R}$ & $\mathrm{LC}$ \\
\hline Bucerotiformes & Bucerotidae & African grey hornbill & Lophoceros nasutus & & $\mathrm{LC}$ \\
\hline Passeriformes & Estrildidae & Red-cheeked cordonbleu & Uraeginthus bengalus & $\mathrm{R}$ & $\mathrm{LC}$ \\
\hline Passeriformes & Estrildidae & Red-billed Firefinch & Lagonosticta senegala & $\mathrm{R}$ & $\mathrm{LC}$ \\
\hline Suliformes & Phalacrocoracidae & $\begin{array}{l}\text { Reed (long-tailes) } \\
\text { cormorant }\end{array}$ & Microcarbo africanus & & $\mathrm{LC}$ \\
\hline Passeriformes & Muscicapidae & Rufous-tailed scrub-robin & Cercotrichas galactotes & $\mathrm{LM} / \mathrm{R}$ & $\mathrm{LC}$ \\
\hline Charadriiformes & Burhinidae & Senegal Thick-knee & Burhinus senegalensis & $\mathrm{R} / \mathrm{LM}$ & $\mathrm{LC}$ \\
\hline Passeriformes & Muscicapidae & Spotted flycatcher & Muscicapa striata & $\mathrm{PM}$ & $\mathrm{LC}$ \\
\hline Charadriiformes & Charadriidae & Spur-winged Lapwing & Vanellus spinosus & $\mathrm{LM} / \mathrm{PM}$ & $\mathrm{LC}$ \\
\hline Pelecaniformes & Ardeidae & Squacco heron & Ardeola ralloides & $\mathrm{AM} / \mathrm{LM}$ & $\mathrm{LC}$ \\
\hline Piciformes & Lybiidae & Vieillot's barbet & Lybius vieilloti & $\mathrm{R}$ & $\mathrm{LC}$ \\
\hline Passeriformes & Viduidae & Village indigobird & Vidua chalybeata & $\mathrm{R}$ & $\mathrm{LC}$ \\
\hline Passeriformes & Ploceidae & Village Weaver & Ploceus cucullatus & $\mathrm{AM} / \mathrm{LM}$ & $\mathrm{LC}$ \\
\hline Cuculiformes & Cuculidae & White browed coucal & Centropus superciliosus & $\mathrm{R}$ & $\mathrm{LC}$ \\
\hline Passeriformes & Motacillidae & White Wagtail & Motacilla alba & PM & $\mathrm{LC}$ \\
\hline Passeriformes & Leiothrichidae & White-headed Babbler & Turdoides leucocephala & $\mathrm{R}$ & $\mathrm{LC}$ \\
\hline Coraciiformes & Meropidae & White-throated Bee-eater & Merops albicollis & $\mathrm{AM} / \mathrm{LM}$ & $\mathrm{LC}$ \\
\hline Charadriiformes & Scolopacidae & Wood Sandpiper & Tringa glareola & PM & $\mathrm{LC}$ \\
\hline Piciformes & Lybiidae & Yellow-breasted barbet & $\begin{array}{l}\text { Trachyphonus } \\
\text { margaritatus }\end{array}$ & $\mathrm{R}$ & $\mathrm{LC}$ \\
\hline Ciconiiformes & Ciconiidae & yelloow-billed storck & Mycteria ibis & LM & $\mathrm{LC}$ \\
\hline Passeriformes & Motacillidae & Yellow Wagtail & Motacilla flava & PM & $\mathrm{LC}$ \\
\hline Pelecaniformes & Ardeidae & Yellow-billed egreat & Ardea brachyrhyncha & $\mathrm{AM} / \mathrm{LM}$ & $\mathrm{LC}$ \\
\hline Passeriformes & Cisticolidae & Zitting cisticola & Cisticola juncidis & $\mathrm{LM} / \mathrm{R}$ & $\mathrm{LC}$ \\
\hline Anseriformes & Anatidae & Hottentot Teal & Spatula hottentota & $\mathrm{AM}$ & $\mathrm{LC}$ \\
\hline Anseriformes & Anatidae & Common teal & Anas crecca & & $\mathrm{LC}$ \\
\hline Anseriformes & Anatidae & Northern Shoveller & Spatula clypeata & PM & $\mathrm{LC}$ \\
\hline Pelecaniformes & Threskiornithidae & Eurasin spoonbill & Platalea leucorodia & PM & $\mathrm{LC}$ \\
\hline
\end{tabular}




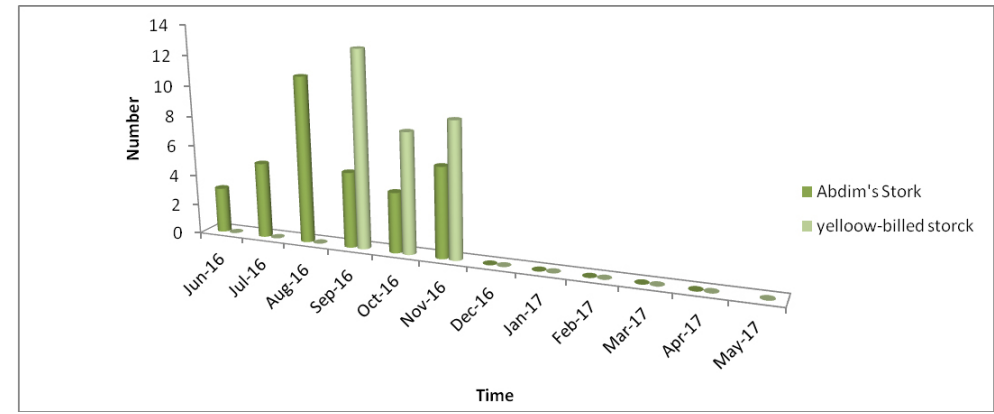

Fig 1. Number of bird's species of the order Ciconiiformes observed in Tuti Island between 2016 -2017.

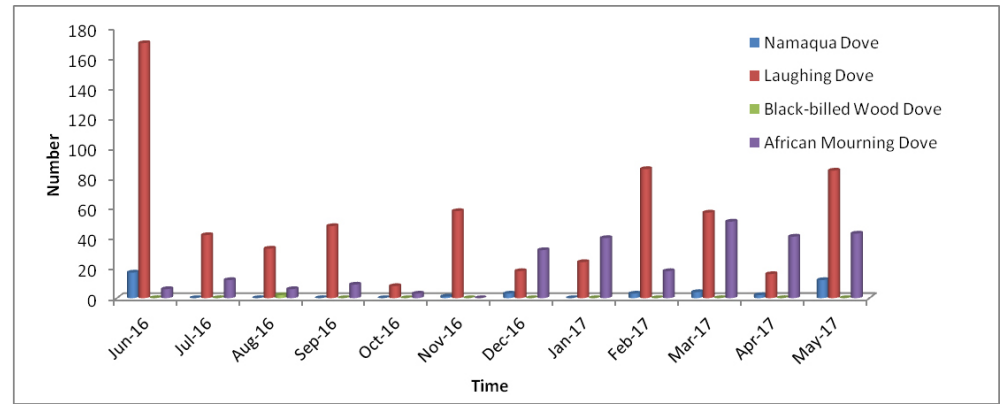

Fig 2. Number of bird's species of the order Columbiformes observed in Tuti Island between 2016 -2017.

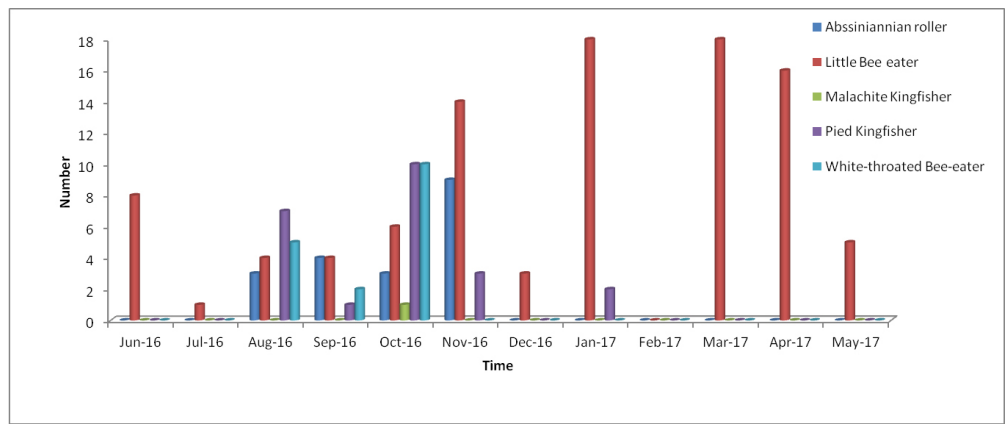

Fig 3. Number of bird's species of the order Coraciiformes observed in Tuti Island between 2016 -2017.

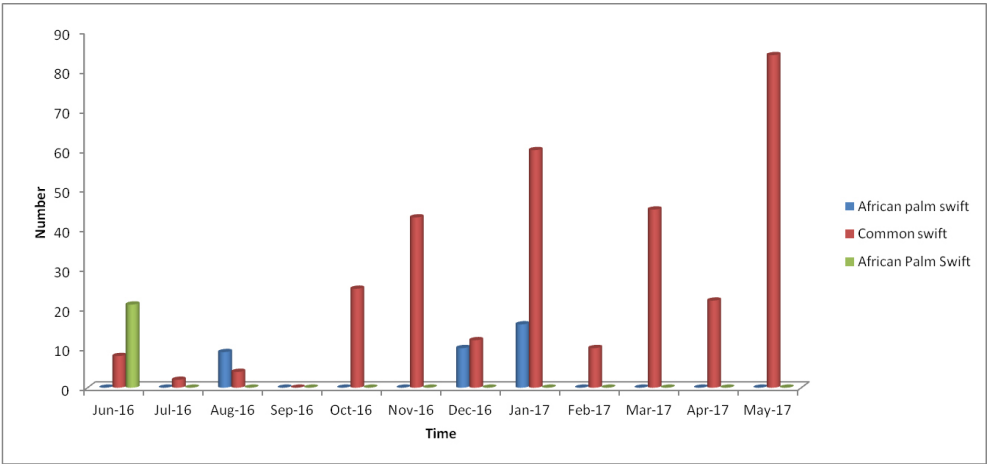

Fig 4. Number of bird's species of the order Apodiformes observed in Tuti Island between 2016 -2017. 


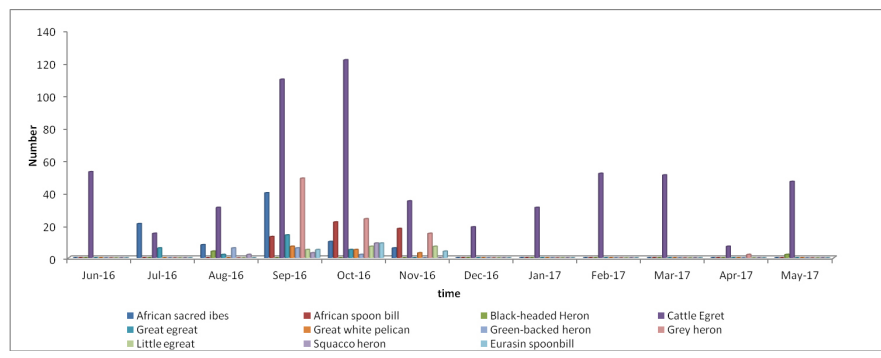

Fig 5. Number of bird's species of the order Pelecaniformes observed in Tuti Island between 2016 -2017.

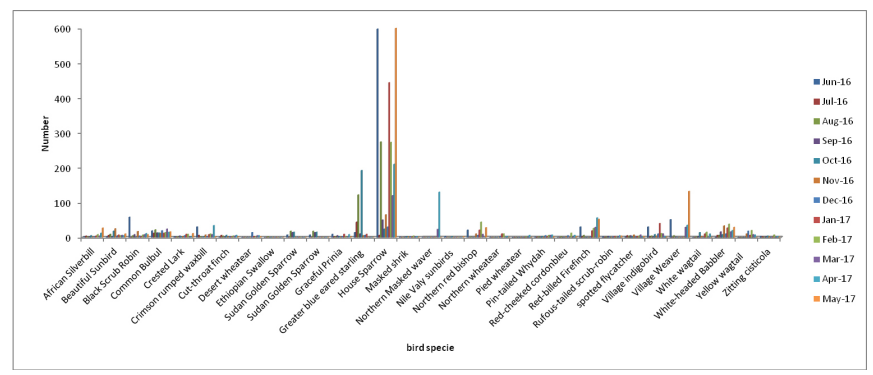

Fig 6. Number of bird's species of the order Passeriformes observed in Tuti Island between 2016 -2017.

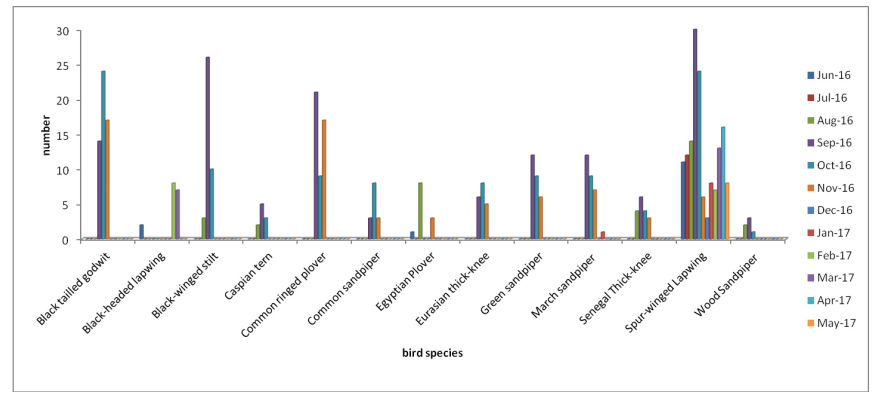

Fig 7. Number of bird's species of the order Charadriiformes observed in Tuti Island between 2016 -2017.

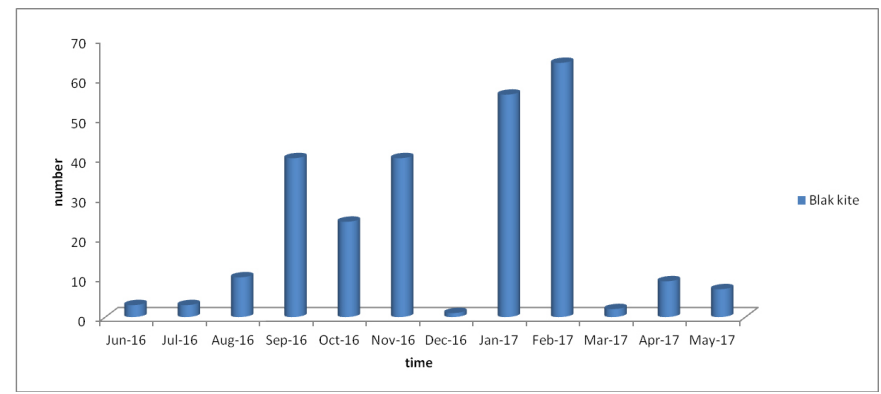

Fig 8. Number of bird's species of the order Accipitriformes observed in Tuti Island between 2016 -2017.

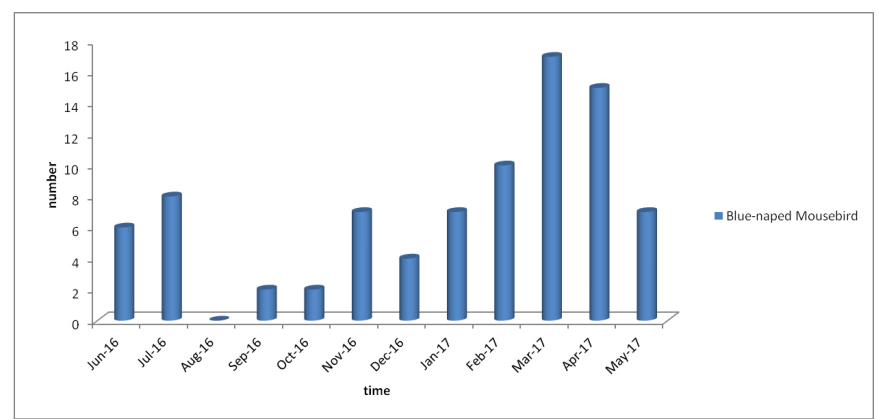

Fig 9. Number of bird's species of the order Coliiformes observed in Tuti Island between 2016 -2017. 


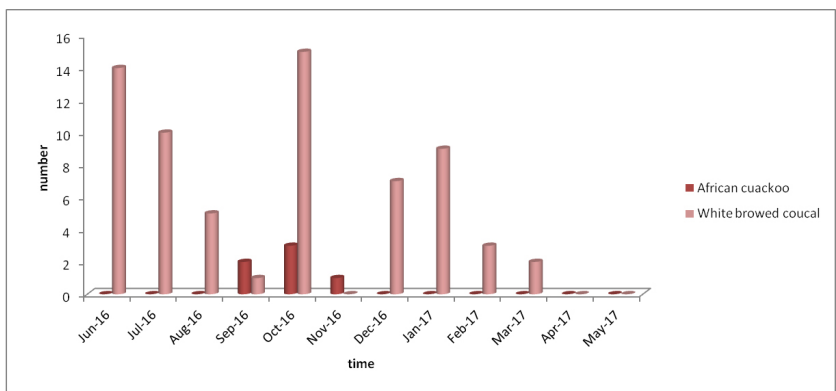

Fig 10. Number of bird's species of the order Cuculiformes observed in Tuti Island between 2016 -2017.

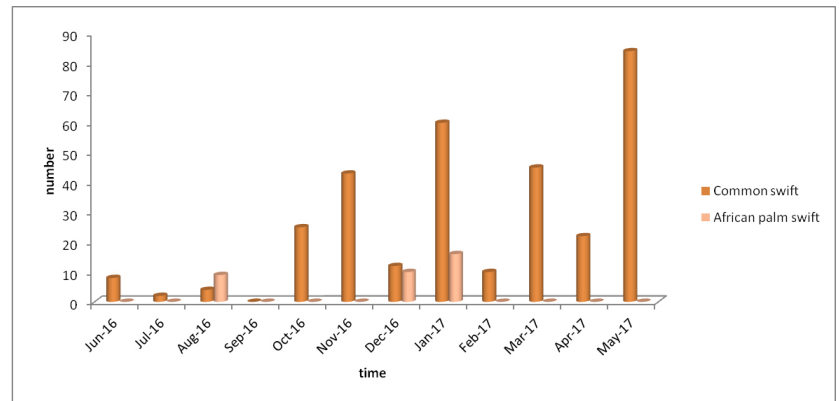

Fig 11. Number of bird's species of the order Apodiformes observed in Tuti Island between 2016 -2017.

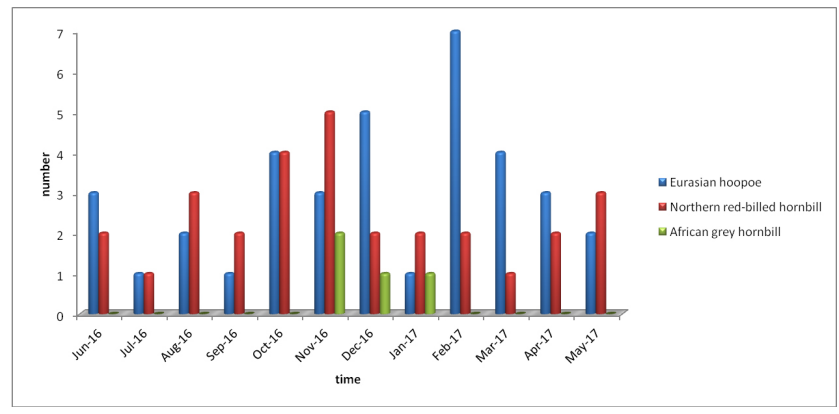

Fig 12. Number of bird's species of the order Bucerotiformes observed in Tuti Island between 2016 -2017.

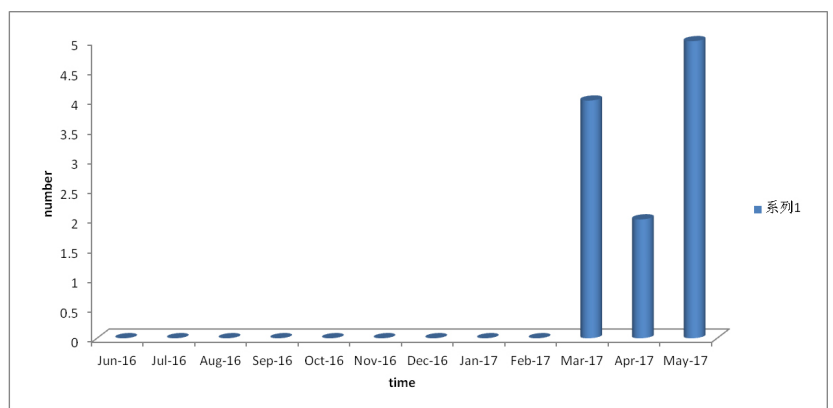

Fig 13. Number of Eurasian Nihgt Gare of the order Caprimulgiformes observed in Tuti Island between 2016 -2017.

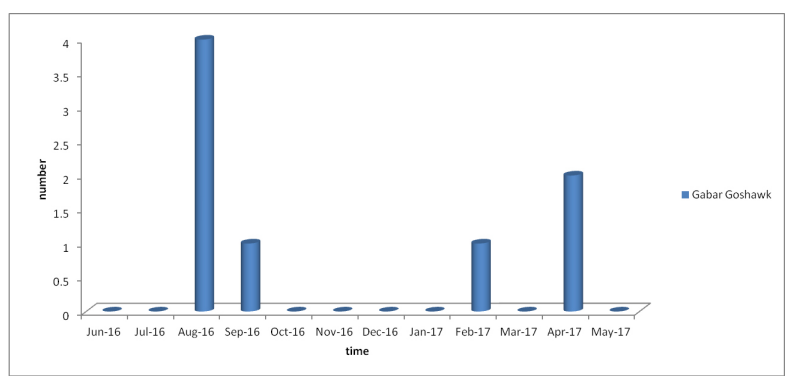

Fig 14. Number of bird species of the order Accipitriformes observed in Tuti Island between 2016 -2017. 


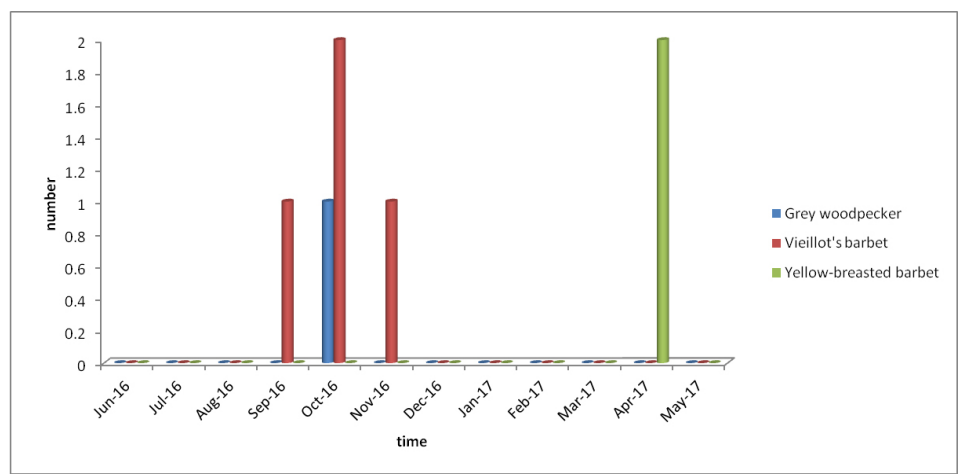

Fig 15. Number of bird species of the order Piciformes observed in Tuti Island between 2016 -2017.

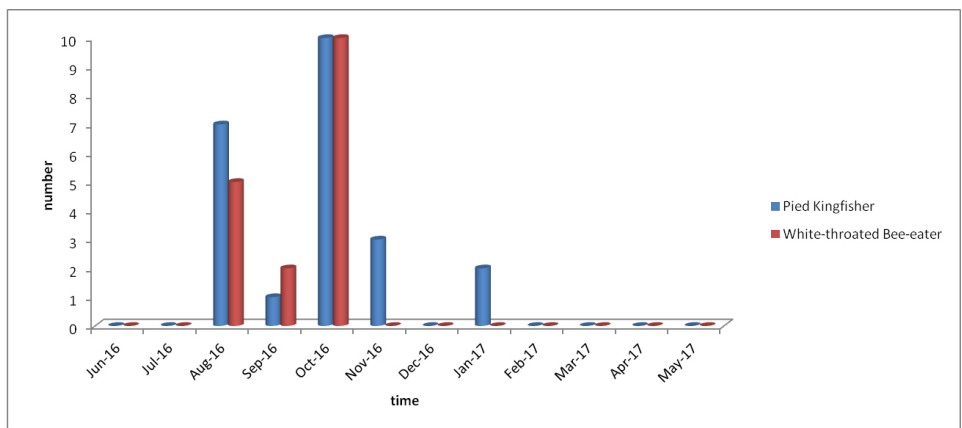

Fig 16. Number of bird species of the order Coraciiformes observed in Tuti Island between 2016 -2017.

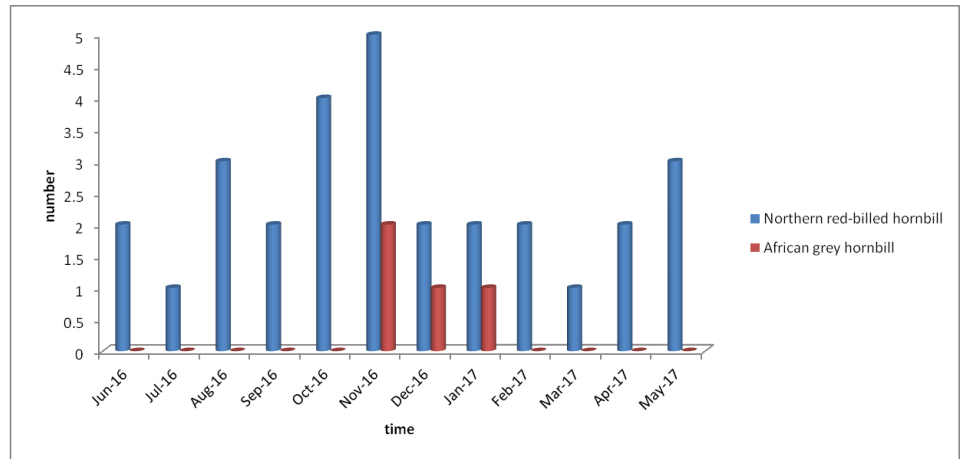

Fig 17. Number of bird species of the order Bucerotiformes observed in Tuti Island between 2016 -2017.

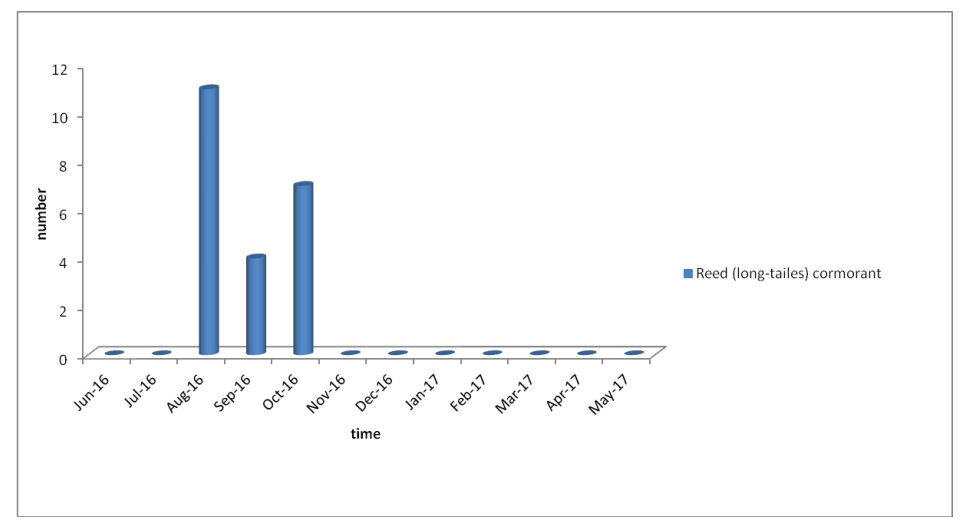

Fig 18. Number of bird species of the order Suliformes observed in Tuti Island between 2016 -2017. 


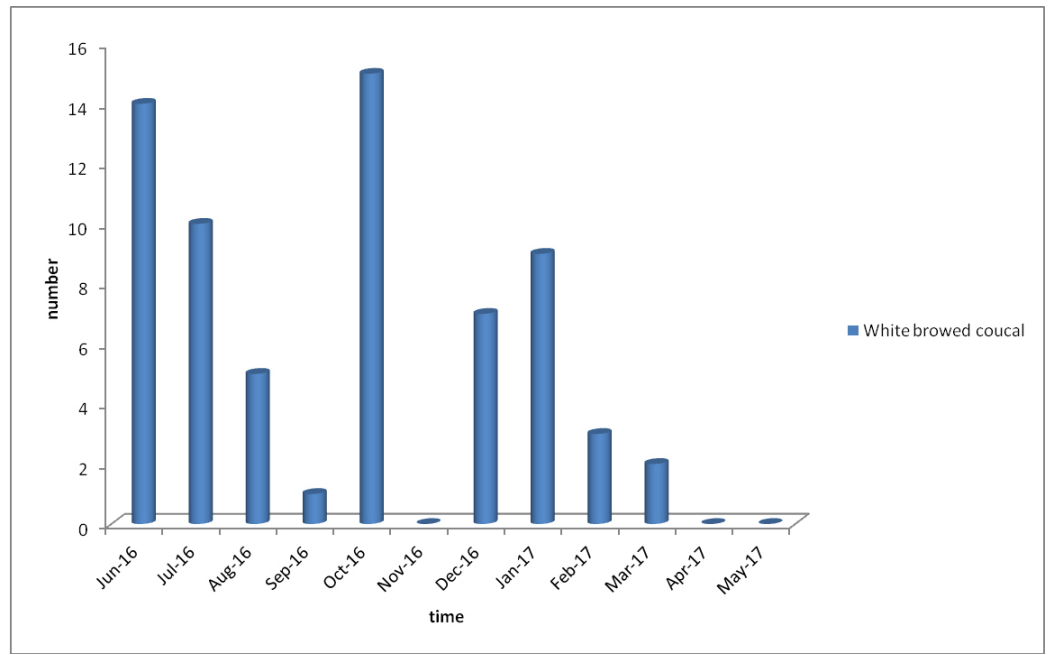

Fig 19. Number of bird species of the order Anseriformes observed in Tuti Island between 2016 -2017.

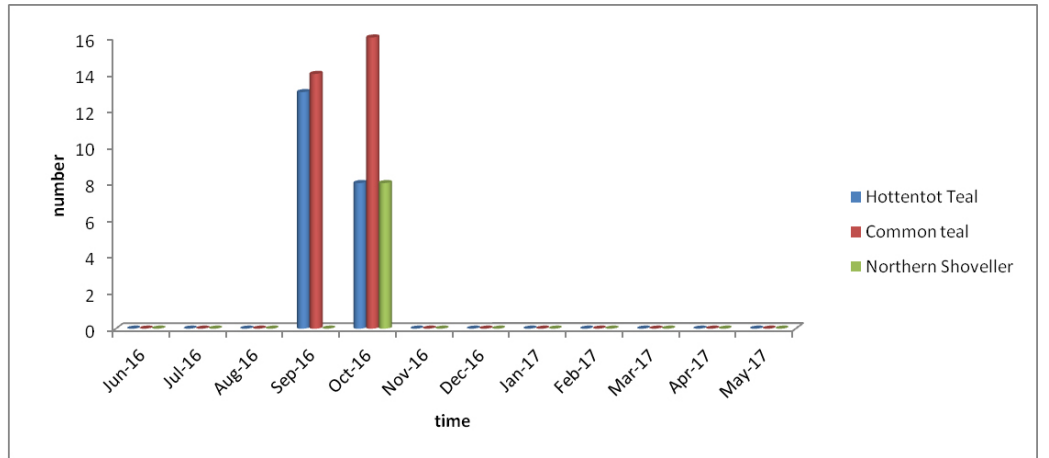

Fig 20. Number of bird species of the order Coraciiformes observed in Tuti Island between 2016 -2017.

\section{Conclusion}

In total, about 8888 individuals that belonged to 88 species, 20 orders were observed in this study, of these 2 species were in the order Ciconiiformes, , 1 species was in order Accipitriformes.

The four most common birds identified were the House Sparrow (Passer domesticus) with 2694 birds identified, followed by the Laughing Dove (Spilopelia senegalensis) with 645 birds identified, the Cattle Egret (Bubulcus ibis) with 573 birds identified and the black Greater blue eared starling (Lamprotornis chalybaeus) with 402 birds identified.

\section{Recommendation}

1. Annual survey of avifauna of Tuti Island is needed to determine the species availability and numbers,

2. Conservation of the island habitat is necessary through the awareness and control of human activities.

In this study it was concluded that A total of 112 species belonging to 17 orders , 27 species were in the order Passeriformes belonging to 12 families (Passeridae, Sylvidae, Ploceidae, Nectariniidae, Motacillidae, Fringilidae, Estrilidae, Corvidae, Laniidae, Sturnidae, Malaconotidae, Muscicapidae and Pycnonotidae). Among the Passeriformes species belonging to 12 families were recorded, of which Ploceidae (weavers, whydahs and sparrows), Sturnidae (starlings and oxpeckers), Estrildidae (waxbills), Laniidae (shrikes), Motacillidae (wagtails) were most frequently observed.

The park have many factors that affect the number, abundance and distribution of birds species, these factors including: availability of water, food resources, vegetation types, suitable nesting sites and nesting materials and other habitat 
component. The various environmental change which take the place in different seasons affect the quality and quantity of food and water which turn reflecting on birds communities. Illegal activities made by local people resident within or in the park edge such as honey collecting set of fire for different purpose and browsing of domestic animals which have been negative effect on birds species this study was conducted in short time, many more species could be identified if the time could be extended in future researches in DNP is rich by birds.

\section{References}

Abdelmageed, S. A. (2008). A Study on the Flora of tuti island in Khartoum state. University of Khartoum. Ali, E. H. (2014). Khartoum New Structure plan and urban Enviornemnt. Khartoum: Sudanes Environemnt Conservation Society.

Benum, L.A. (1999). Threatened birds and rural communities: Balancing the equation. In: Proc. 22 International Ornithology Congress. Durban: pp 1546-- - 1555 (Ed. By N.J. Adams \& R.H. Slotow). BirdLife South Africa, Johannesburg.

Davies, H. R. . (1994). A Rural “Eye” in the Capital : Tuti Island. GeoJournal, 1994(1949), 387-392.

Ibrahim, M. B., \& Davies, H. R. . (1991). Tuti Island: A rural system in an Urban Locality. In M. E. A. Sin \& H. R.

Davies (Eds.), The Future of Sudan's Capital region: A study in the Development and Change. Khartoum University Press.

Furness, R.W. \& Greenwood, J.J.D. (Eds.) (1993). Birds as Monitors of Environmental Change. Chapman \& Hall, London, UK.

Starkley, M. Birnie, N., Cameron, A., Daffa, R. A., Haddelsey, L., Hood, L., Johnson, N., Kapapa, L., J., Mwangomo, E., Rainey, H. \& Robinson, W. (2002). Kilombero Wildlife Project - An ecological and social survey in the Kilombero Valley, Tanzania. Kilombero Wildlife Project, Edinburgh.

Eltyeb, H., Idris, E., Adam, A.Ezalden, T. and Hamed, D(2012). A forest in city: Biodiversity at Sunut forest, Khartoum, Sudan. The Egyptian Academic journal of biological sciences. Zoology, 4:199-209. 